\title{
MULTI-LEVEL MODELS FOR LONGITUDINAL GROWTH NORMS
}

\author{
HUIQI PAN* AND HARVEY GOLDSTEIN \\ Institute of Education, 20 Bedford Way, London, WC1H OAL, U.K.
}

\begin{abstract}
SUMMARY
Multi-level models for estimating conditional and unconditional longitudinal growth norms are presented. The procedure involves transforming the original growth measurements to Normality and modelling these with a two-level random coefficient model. Growth norms for any desired time interval and function can be derived. Height and weight data are used for illustration. (C) 1997 by John Wiley \& Sons, Ltd.
\end{abstract}

Statist. Med., 16, 2665-2678 (1997)

No. of Figures: 9 No. of Tables: $5 \quad$ No. of References: 19

\section{INTRODUCTION}

Tanner et al. ${ }^{1}$ suggested the use of two types of norms for monitoring a child's growth, distance or size norms for growth attained and velocity norms for rate of growth. The norms typically are conditional on gender, age and nationality. To obtain more precision one may include extra variables. For example, Tanner et al. ${ }^{2}$ published norms for British children's height at ages 2 to 9 allowing for the height of parents. Tanner et al. ${ }^{3}$ considered the birthweight of siblings in norms for birthweight.

Healy ${ }^{4}$ suggested a regression approach for measurements at age $t$ conditional on age one year previously. The regression approach has been adopted by several authors. Cameron ${ }^{5}$ published conditional norms for growth in height of British children from 5.0 to 15.99 years of age. Berkey et $a l{ }^{6}$ presented conditional norms for length and weight in pre-school children of the Harvard Longitudinal Study in the U.S.A. Means, standard deviations and correlations at ages one year apart were provided for clinical application. With these statistics, and assuming Normality, conditional norms involving two given measurements can be derived. ${ }^{6} \mathrm{Cole}^{7}$ presented conditional height velocity charts for children from the age of 1 month to 19 years using data from a French longitudinal study. He provided estimates of regression coefficients for measurements at ages one year apart and also for measurements two years apart. He extended the conditional norms for measurements that are not normally distributed by using transformed $(z)$ scores which were obtained by the LMS method. ${ }^{7,8}$ As Healy ${ }^{4}$ has noted, the conditional norms show greater sensitivity than velocity norms and avoid wrongly ascribing abnormally low velocities in situations of high initial measurement.

* Correspondence to: H. Pan, Institute of Education, 20 Bedford Way, London, WC1H 0AL, U.K. 
The principal drawback of both velocity and conditional norms is that they are based upon measurements made over predefined time intervals, typically either 1.0 or 2.0 years. This constraint makes them more complicated to use than the conventional growth chart especially in a clinic setting.

\section{Norms for longitudinal data}

In practical clinical situations, children will often present themselves at irregular intervals and may have three or more serial measurements. In the present paper we develop a procedure which allows children to be judged against norms appropriate to any number of serial measurements at an arbitrary set of ages. Before describing the procedure in detail we note two important consequences of having such a procedure. First, the complexity involved rules out the use of simple growth charts; the procedure requires computer software with which clinicians can interact. Secondly, when more than two serial measurements are involved, there are many possible ways of summarizing growth for the purposes of comparing a child against population standards. Thus, for example, with three serial measurements we could calculate an overall 'acceleration' or we could use the distribution of the last measurement conditional on the previous two, or we could calculate an average velocity. Indeed, we may wish to do all of these, but this does rise the issue of how we should characterize growth in such circumstances. The present paper does not attempt a full discussion of this issue, but we shall present examples and different characterizations and show that these lead to new ways of studying growth patterns.

For age related measurements Cole ${ }^{9}$ suggested using a Box-Cox transformation to smoothly transform a set of measurements to Normality by grouping and modelling the relationship of the variation and the skewness with age. Although originally proposed for cross-sectional data it can be applied to longitudinal data also. We review briefly this method, known as LMS, as developed subsequently by Cole and Green ${ }^{8}$ with the aim of producing transformed scores which, with respect to age, are conditionally Normal and unconditionally, for any given set of ages, have a multivariate Normal distribution. Another approach is that of Royston. ${ }^{10}$ The second stage of the procedure is to model this multivariate distribution as a two-level repeated measures structure. The third stage considers the distributions of the summary statistics required to establish longitudinal norms. An alternative single-stage approach ${ }^{11}$ is to construct a non-linear model for the original measurements which incorporates parameters which effectively define a Normalizing transformation. It is possible, in principle, to extend this approach to our situation, but there are considerable practical difficulties and we shall not consider this possibility further.

\section{METHODS}

\section{Stage 1: Generating Normalized scores}

The LMS method attempts to Normalize data by modelling the median, the coefficient of variation and the Box-Cox power curve to remove skewness from the data, as smooth functions of age, $M(t), S(t)$ and $L(t)$. Maximum penalized likelihood estimation is used for the estimates of $M(t), S(t)$ and $L(t)$, which are cubic splines with knots at each distinct value of $t$. The three quantities provide the required norms $C_{100 \alpha}$ at age $t$ by inserting the appropriate Normal equivalent deviate $d_{\alpha}$ for tail area $\alpha$, in the equation

$$
C_{100 \alpha}(t)=M(t)\left[1+L(t) S(t) d_{\alpha}\right]^{1 / L(t)}
$$


The equivalent score $(z)$ in standard deviation units corresponding to a measurement $y$ of a child thus can be obtained using the equation

$$
z=\left\{[y / M(t)]^{L(t)}-1\right\} /[L(t) S(t)] .
$$

We refer to $z$ as an 'empirical $L M S$ ' $(E L M S)$ score. It corresponds to the standard deviation or ' $z$ ' score commonly used in the reporting of growth data.

\section{Stage 2: Fitting a two-level model to the Normalized scores}

If we assume that the LMS procedure provides Normally distributed scores then, using the $E L M S$ score $z_{i j}$ as response, we can construct a two-level model

$$
\begin{gathered}
z_{i j}=\sum_{h=0}^{p} \beta_{h} t_{i j}^{h}+\sum_{h=0}^{q} u_{h j} t_{i j}^{h}+e_{i j} \\
E=\left\{e_{i j}\right\} \sim \mathrm{N}\left(0, \sigma_{e}^{2} I\right) \\
U=\left\{u_{h j}\right\}=\mathrm{N}\left(0, D_{u}\right) \\
i=1, \ldots, n_{j} ; \quad j=1, \ldots, m
\end{gathered}
$$

where $n_{j}$ is the number of level 1 units (measurement occasions) within the $j$ th individual and $m$ is the number of individuals. There are $p$ fixed coefficients and $q$ random coefficients. The first summation on the right hand side of (3) is the 'fixed' part of the model which is this case represents the average polynomial trend with age. We shall explore the Normality assumption in (3) further in the example we use to illustrate the procedure. In model (3) we assume that the level 1 residuals $e_{i j}$ are distributed independently with constant variance. This latter assumption can be verified empirically and if necessary modified by fitting a model where, for example, the variance is allowed to depend on time (reference 12, Chapter 3). The independence assumption is likely to be violated where measurements are taken close together in time. For example, Goldstein et al. ${ }^{13}$ show that for height measurements made 3 months apart at the start of puberty, an autoregressive model is required. It is possible to extend (3) to include a dependency structure among the level 1 residuals and to incorporate the additional parameters in the construction of norms, but we shall not pursue this in the present paper.

It should be emphasized that modelling on the scale of the $z_{i j}$ is statistically convenient since it allows us to characterize fully the multivariate data structure.

\section{Stage 3: Establishing the norms}

Using the final model parameter values we can calculate any required functions of the $z_{i j}$ and estimate their population distribution. We are not restricted to a particular set of ages or number of measurements since, in principle, the joint distribution of any set of $z_{i j}$ is entirely determined from (3). We note that while the modelling can be based upon longitudinal data covering a wide age range, norms are typically required for serial measurements over a relatively restricted age range and this is reflected in the following discussion and examples. 


\section{Conditional norms}

Suppose we have three serial measurements on a subject and we wish to provide norms for the third measurement conditional on the first two. We standardize the $z_{i j}$ in (3) to have zero mean by subtracting the fixed part of (3). Then, for a measurement $z_{3 j}$ at time $t_{3}$ with a measurement $z_{2 j}$ at previous line $t_{2}$ and a further measurement $z_{1 j}$ at $t_{1}, j=1,2, \ldots, m$, the conditional relationship can be written as the linear regression

$$
z_{3 j}=\beta_{1} z_{1 j}+\beta_{2} z_{2 j}+\varepsilon_{j}
$$

where the residual $\varepsilon_{j}$ is assumed to be distributed as $\mathrm{N}\left(0, \sigma_{\varepsilon}^{2}\right)$. We have $E\left(z_{1 j}\right)=E\left(z_{2 j}\right)=0$. Let $Z_{i}$ be a random vector of dimension $m \times 1$ containing the $z_{i j}$. We have

$$
Z=\left\{Z_{i}\right\} \sim \mathrm{N}(0, D)
$$

where $D$ is a $3 \times 3$ convariance matrix determined by the parameters of the two-level model (3) and the values of the $\left\{t_{i}\right\}$.

We define the standardized, $\mathrm{N}(0,1)$, variable corresponding to $z_{3 j}$ for the $j$ th child conditional on $z_{1 j}$ and $z_{2 j}$ as

$$
z_{3 j}^{*}=\frac{z_{3 j}-\beta_{1} z_{1 j}-\beta_{2} z_{2 j}}{\sigma_{\varepsilon}}
$$

where $\sigma_{\varepsilon}^{2}$ is the residual variance obtained from (4). On substituting the observed (transformed) measurements we obtain the required standardized values or the equivalent percentiles.

\section{Unconditional norms}

Over a short time interval, growth rate can be taken to be constant and it can be estimated by the slope of the regression of two or more successive measurements on age. The slope will be a linear function of the measurements with coefficients depending upon the ages and its distribution can be obtained readily from the model parameter estimates. For longer intervals a quadratic can be used, the first and second derivatives providing estimates of velocity and acceleration. Again, these will be linear functions of the observed measurements. More generally, non-linear functions may be needed. Given the model parameters, the distributions of such quantities are most easily obtained by straightforward simulation, though many replicates will be needed to obtain precise estimates of extreme percentiles.

It would also be possible to produce conditional velocity (or acceleration) norms by regressing, say, the difference between two successive measurements on a set of previous measurements. There would seem to be little advantage in this, however, over the conditional norms described above.

\section{Bivariate norms}

The construction of norms for weight allowing for height has been much discussed. ${ }^{14}$ We can use our methodology to construct norms for weight conditional on current height, together with previous measurements of height and weight if required. For this purpose we need a bivariate response repeated measure model. We may write such a model as an extension of (3), 
Table I. Numbers of measurements of height and weight for 91 boys

\begin{tabular}{cccccc}
\hline Age & Height & Weight & Age & Height & Weight \\
\hline $2 \cdot 0+$ & 75 & 75 & $10 \cdot 5+$ & 84 & 84 \\
$2 \cdot 5+$ & 76 & 76 & $11 \cdot 0+$ & 86 & 85 \\
$3 \cdot 0+$ & 90 & 90 & $11 \cdot 5+$ & 83 & 82 \\
$3 \cdot 5+$ & 86 & 86 & $12 \cdot 0+$ & 82 & 82 \\
$\cdot 0+$ & 89 & 87 & $12 \cdot 5+$ & 85 & 85 \\
$4 \cdot 5+$ & 82 & 82 & $13 \cdot 0+$ & 83 & 83 \\
$5 \cdot 0+$ & 87 & 87 & $13 \cdot 5+$ & 71 & 70 \\
$5 \cdot 5+$ & 84 & 83 & $14 \cdot 0+$ & 85 & 83 \\
$6 \cdot 0+$ & 81 & 81 & $14 \cdot 5+$ & 83 & 83 \\
$6 \cdot 5+$ & 87 & 86 & $15 \cdot 0+$ & 80 & 79 \\
$7 \cdot 0+$ & 88 & 88 & $15 \cdot 5+$ & 79 & 78 \\
$7 \cdot 5+$ & 86 & 84 & $16 \cdot 0+$ & 81 & 81 \\
$8 \cdot 0+$ & 84 & 84 & $16 \cdot 5+$ & 75 & 74 \\
$8 \cdot 5+$ & 83 & 83 & $17 \cdot 0+$ & 65 & 65 \\
$9 \cdot 0+$ & 91 & 91 & $17 \cdot 5+$ & 56 & 55 \\
$9 \cdot 5+$ & 83 & 83 & $18 \cdot 0$ & 34 & 34 \\
$10 \cdot 0+$ & 89 & 88 & & & \\
\hline
\end{tabular}

namely

$$
z_{i j}=\delta\left[\sum_{h=0}^{p} \beta_{h} t_{i j}^{h}+\sum_{h=0}^{q} u_{h j} t_{i j}^{h}+e_{i j}\right]+(1-\delta)\left[\sum_{h=0}^{p^{\prime}} \beta_{h}^{\prime} t_{i j}^{h}+\sum_{h=0}^{q^{\prime}} u_{h j}^{\prime} t_{i j}^{h}+e_{i j}^{\prime}\right]
$$

where the prime (') denotes the random variables associated with weight. The response vector consists of the complete set of weight and height measurements, hierarchically ordered so that the two measurements are nested within each measuring occasion and measuring occasions are nested within subjects. The indicator variable $\delta$ is 1 if the response is a weight measurement and 0 if height. From this model we can estimate the joint distribution of height and weight measurements and thus derive norms for any function of these measurements as before. For a more detailed description of this model, and the more general multivariate one, and how they can be fitted within a repeated measures structure, see Goldstein (reference 12, Chapter 6).

\section{EXAMPLES}

The data used to illustrate the methodology consist of 91 males from the Edinburgh Longitudinal Study, initiated in 1972. These are known to be chromosomally normal as they were born at a time when the Medical Research Council was conducting a new-born cytogenetic survey. ${ }^{15}$ The children were measured 3-monthly during the first year of life and twice-yearly thereafter. The data used to illustrae the procedure in the present paper cover ages from 2 to 18.5 years. The number of measurements in each age group is given in Table I. We present separate results for height and weight and for a bivariate modelling of weight and height.

The ELMS scores were computed using software provided by Dr. T. Cole. Using these scores, Table II gives the two-level model estimated with the MLn software package. ${ }^{16}$ Various models with different values of $p$ and $q$ in (3) and (7) were explored using standard likelihood ratio tests to 
Table II. A two-level model for ELMS scores of height and weight (standard errors in brackets)

\begin{tabular}{|c|c|c|c|c|c|}
\hline \multirow[b]{2}{*}{ Random level 2} & \multirow[b]{2}{*}{$u_{0}$} & \multicolumn{2}{|c|}{ Covariance matrix } & \multirow[b]{2}{*}{$u_{3} \times 10^{3}$} & \multirow[b]{2}{*}{$u_{4} \times 10^{4}$} \\
\hline & & $u_{1} \times 10$ & $u_{2} \times 10^{2}$ & & \\
\hline \multicolumn{6}{|c|}{ Height: Intercept 0.034(0.092) } \\
\hline$u_{0}$ & $0.96(0 \cdot 14)$ & & & & \\
\hline$u_{1} \times 10$ & $0.06(0.08)$ & $0 \cdot 57(0 \cdot 09)$ & & & \\
\hline$u_{2} \times 10^{2}$ & $-0 \cdot 18(0 \cdot 13)$ & $0 \cdot 26(0 \cdot 11)$ & $1 \cdot 39(0 \cdot 24)$ & & \\
\hline$u_{3} \times 10^{3}$ & $0.51(0 \cdot 25)$ & $-1 \cdot 39(0 \cdot 24)$ & $-0.67(0 \cdot 32)$ & $5 \cdot 12(0 \cdot 81)$ & \\
\hline$u_{4} \times 10^{4}$ & $-0.50(0.28)$ & $1.29(0.26)$ & $-1.88(0.43)$ & $4.95(0.86)$ & $6.49(1.08)$ \\
\hline Variance level 1 & $0.019(0 \cdot 0006)$ & & & & \\
\hline \multicolumn{6}{|c|}{ Weight: Intercept 0.037(0.089) } \\
\hline$u_{0}$ & $1.00(0.15)$ & & & & \\
\hline$u_{1} \times 10$ & $0.08(0.09)$ & $0.74(0.12)$ & & & \\
\hline$u_{2} \times 10^{2}$ & $-0.79(0.22)$ & $-0.45(0 \cdot 19)$ & $3 \cdot 36(0 \cdot 57)$ & & \\
\hline$u_{3} \times 10^{3}$ & $-0.03(0.13)$ & $-0.68(0.14)$ & $0 \cdot 35(0 \cdot 25)$ & $1 \cdot 18(0 \cdot 22)$ & \\
\hline$u_{4} \times 10^{4}$ & $0.28(0.29)$ & $0.35(0.25)$ & $-3.84(0.73)$ & $-0.29(0.34)$ & $5.41(1.04)$ \\
\hline Variance level 1 & $0.036(0.001)$ & & & & \\
\hline
\end{tabular}

Age is centred at 11 years. At level $2 u_{i}$ is the coefficient of the polynomial term of order $i$

choose a final model which was an adequate fit to the data. No serious convergence problems were experienced during the fitting of these models.

These two-level models show that the means of the normalized scores are not significantly different from zero so that no fixed part is in fact needed in these models. As a check the estimates of the standard deviation of the ELMS scores by age have been compared with those obtained directly using standardized scores for each one year age group. The difference between the estimates within age groups is small, being no greater than 6 per cent. We have also studied the year on year correlations based on the ELMS scores and these correspond closely to those reported by other studies. ${ }^{5-7,14,17}$ We note, however, that the ELMS scores are transformations of the raw scores. Since the raw scores tend to have skewness, transformation to Normality will tend to decrease the correlations, which is what occurs. Equation (2) or a similar conversion formula can be used to convert measurements to ELMS scores or vice versa.

\section{Using the norms}

To illustrate the methodology we shall consider sample measurements approximately one year apart but the procedure is quite general (see discussion).

We have chosen a tall boy (number 303) for whom we wish to calculate a standardized score for a measurement at age 11.65 years conditional on the previous three measurements and also velocity and acceleration estimates for the same period. Table III gives his age, height measurements and corresponding ELMS scores, the final one lying above the 99th centile.

In Table IV standardized scores for conditional norms, average velocity and average acceleration are given, with clear differences being apparent. Conditional norms can also be presented on the original scale of height using (1), and Table $\mathrm{V}$ illustrates the use of this conversion by presenting the percentile in terms of height at 11.65 years, conditional on one, two or three year previous measurements. 
Table III. Data for boy number 303

\begin{tabular}{rcc}
\hline Age & Height $(\mathrm{cm})$ & ELMS \\
\hline $8 \cdot 50$ & $152 \cdot 7$ & $3 \cdot 40$ \\
9.51 & $157 \cdot 9$ & $3 \cdot 27$ \\
$10 \cdot 52$ & $162 \cdot 7$ & $3 \cdot 14$ \\
11.65 & $167 \cdot 7$ & $2 \cdot 91$ \\
\hline
\end{tabular}

Table IV. Standardized scores for the height at 11.65 years in Table III

\begin{tabular}{lcc}
\hline Number of previous measurements & Value & Percentile \\
\hline Conditional norm & & \\
One & $-0 \cdot 72$ & 24 \\
Two & $-1 \cdot 28$ & 10 \\
Three & $-1 \cdot 18$ & 12 \\
Velocity & & \\
One & $-1 \cdot 11$ & 13 \\
Two & $-1 \cdot 44$ & 7 \\
Three & $-1 \cdot 74$ & 4 \\
Acceleration & & \\
Two & $-0 \cdot 24$ & 41 \\
Three & $-0 \cdot 29$ & 39 \\
\hline
\end{tabular}

Table V. Conditional centiles $(\mathrm{cm})$ for the height at 11.65 years in Table III

\begin{tabular}{lccccc}
\hline $\begin{array}{l}\text { Number of previous } \\
\text { measurements }\end{array}$ & 3 & 10 & 50 & 90 & 97 \\
\hline One & $165 \cdot 9$ & $166 \cdot 9$ & $168 \cdot 9$ & $170 \cdot 9$ & $171 \cdot 8$ \\
Two & $166 \cdot 8$ & $167 \cdot 7$ & $169 \cdot 7$ & $171 \cdot 6$ & $172 \cdot 5$ \\
Three & $166 \cdot 7$ & $167 \cdot 6$ & $169 \cdot 5$ & $171 \cdot 5$ & $172 \cdot 4$ \\
\hline
\end{tabular}

From the sample we can estimate standardized scores for an individual for all of his measurements. This provides a new way of studying growth patterns in terms of changes of velocity, acceleration and conditional values with age. Figures 1 and 2 give the relevant plots for subject number 303, where the standardized scores of velocities and accelerations are plotted at the ages in the centre of the time interval. We find that the acceleration estimates tend to move across centiles earlier than for the corresponding velocities. The differences for average velocities and accelerations based on two, three or four measurements are also clear.

We can also illustrate contrasts among growth patterns by studying subjects at different overall growth positions. Figures 3-5 give the conditional plots using one, two or three prior measurements against age for cases 303, 249 and 241, who are tall, median and short individuals, 


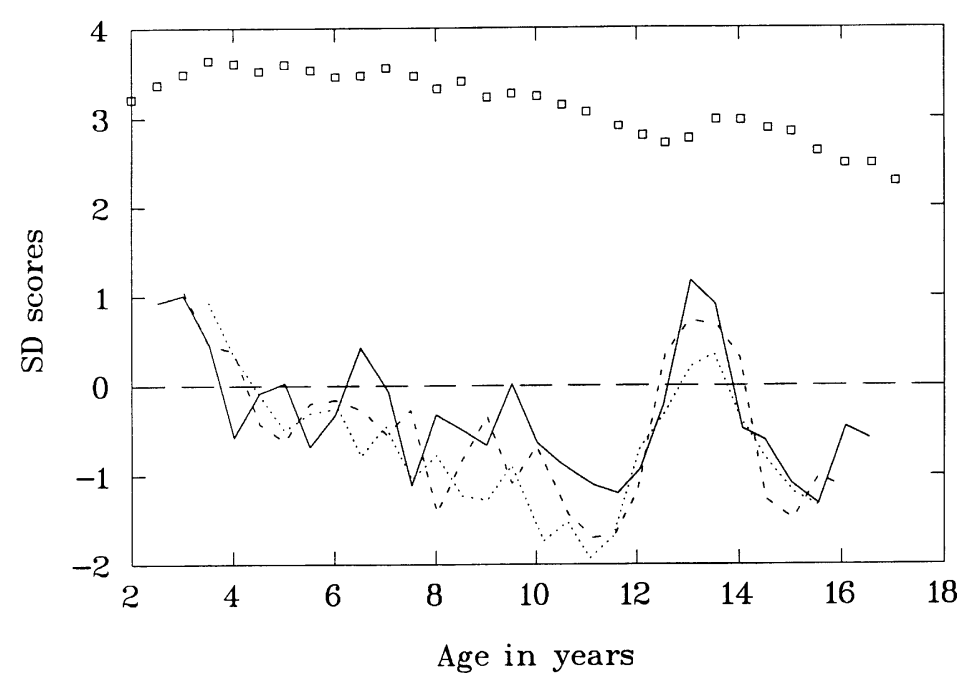

Figure 1. Standardized scores of average velocity of height for child 303 $\square \quad$ ELMS scores of height

- for two occasions

- - - for three occasions

$\ldots$ for four occasions

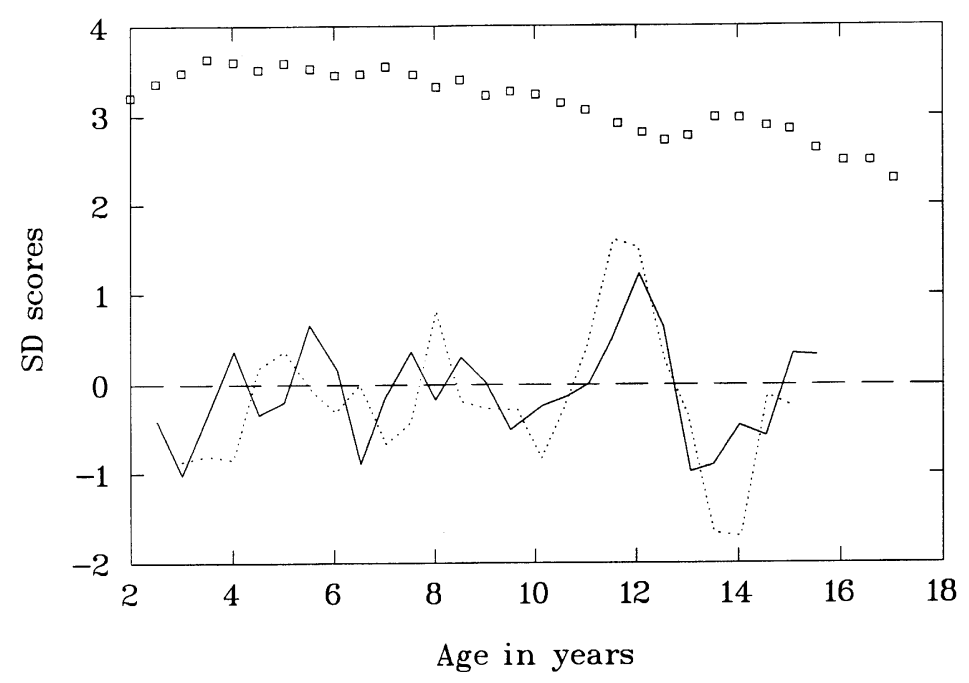

Figure 2. Standardized scores of average acceleration of height for child 303 $\begin{array}{ll}\square \quad & \begin{array}{l}\text { ELMS scores of height } \\ \text { for three occasions }\end{array} \\ \cdots \cdots & \text { for four occasions }\end{array}$ 


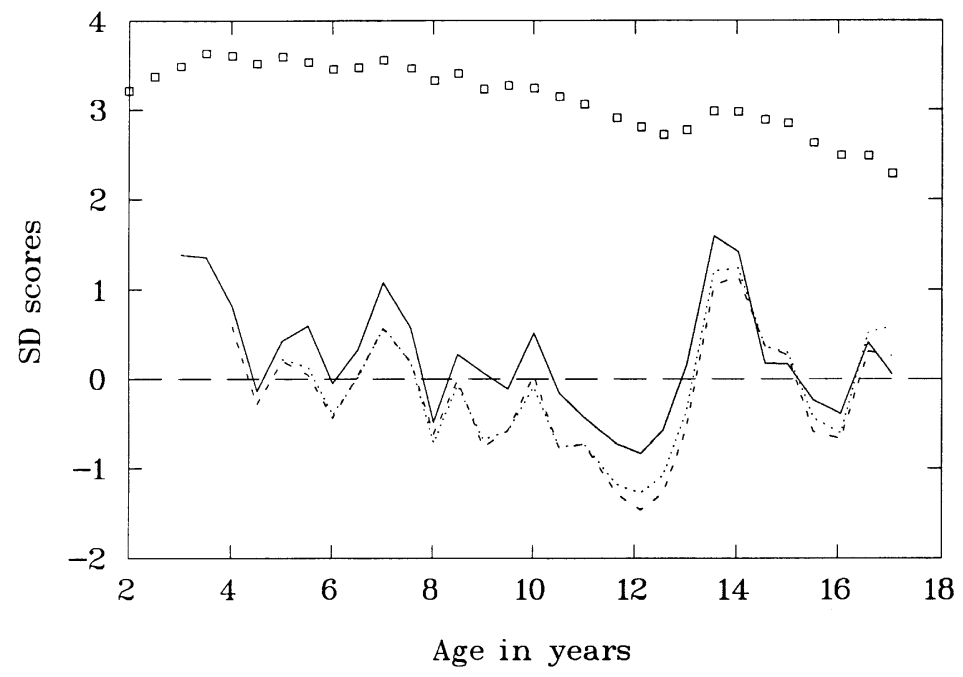

Figure 3. Standardized scores of conditional norms of height for child 303

$\square \quad$ ELMS scores of height

_ conditional on height one year earlier

---- conditional on height one and two years earlier

$\cdots$ conditional on height one, two and three years earlier

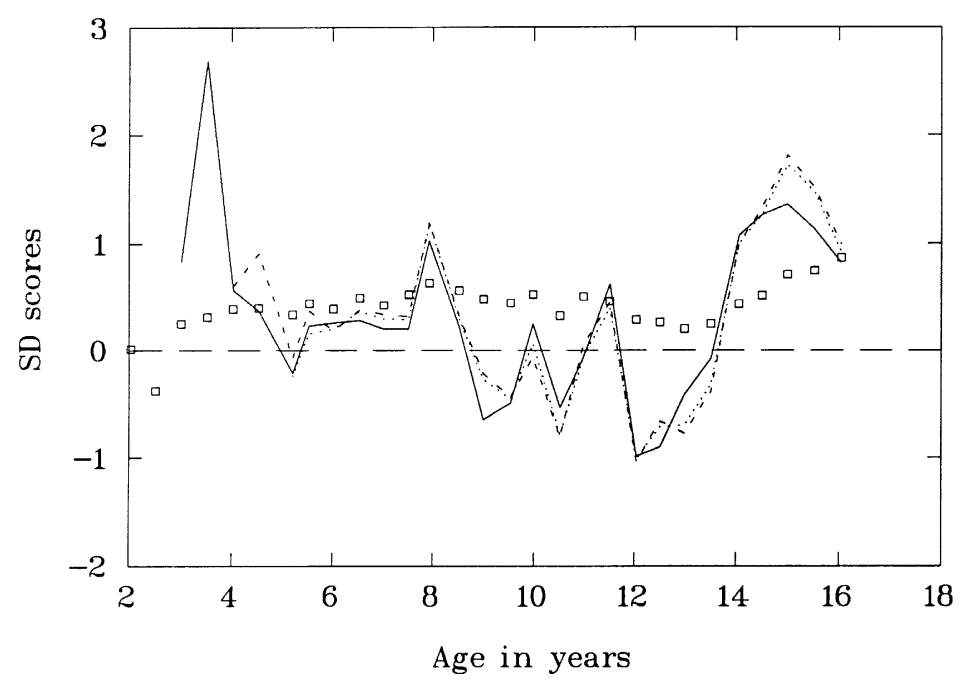

Figure 4. Standardized scores of conditional norms of height for child 249

$\square \quad$ ELMS scores of height

_ conditional on height one year earlier

- - - conditional on height one and two years earlier

.... conditional on height one, two and three years earlier 


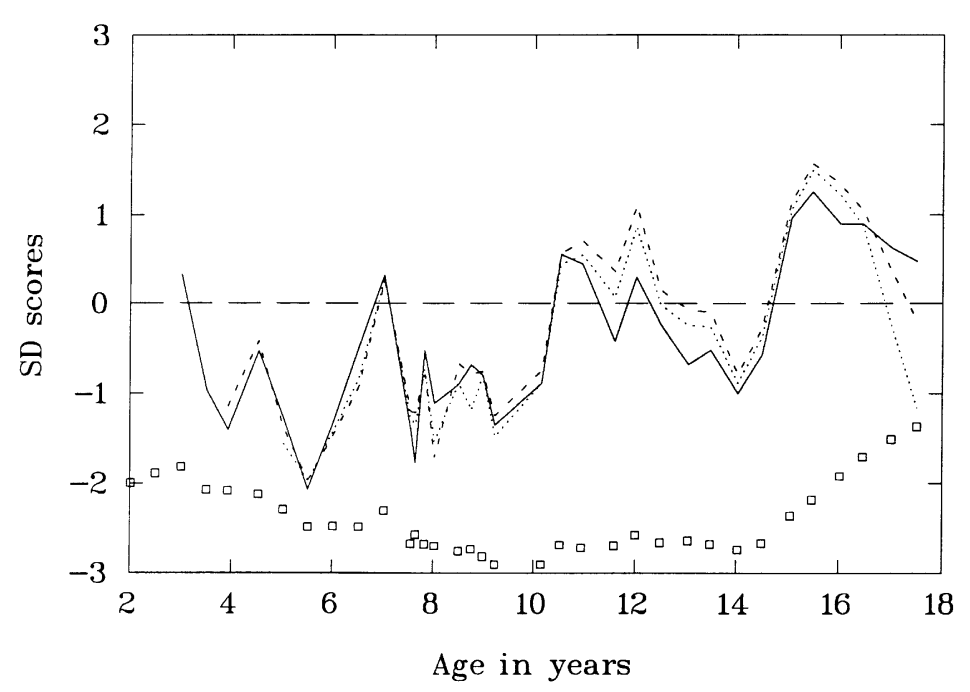

Figure 5. Standardized scores of conditional norms of height for child 251

$\begin{array}{ll}\square \square & \begin{array}{l}\text { ELMS scores of height } \\ \text { conditional on height one year earlier }\end{array} \\ ---- & \begin{array}{l}\text { conditional on height one and two years earlier } \\ \text { conditional on height one, two and three years earlier }\end{array}\end{array}$

respectively. The plots show three peaks, located at about 7,10 and 14 years. A similar picture emerges for weight (see Figure 6).

To illustrate the use of bivariate norms, Figure 7 contrasts the ELMS scores of weight and height with that for weight conditional on current height for subject 303 by age. Figure 8 plots the standardized scores for weight, conditional on weight one and two years previously; and for weight conditional on weight one and two years previously together with current height and with current height and height one year previously. It appears that the conditional weight norms are little changed by the addition of height.

We have studied the distribution of standardized residuals ${ }^{12}$ for the scores at levels 1 and 2 and Normal score plots do not show obvious violations of the model assumptions. Figure 9 shows three of these Normal score plots which are typical.

\section{DISCUSSION}

The main purpose of this paper is to introduce a new methodology for constructing longitudinal growth norms, to apply this to an existing data set and to illustrate new ways of studying growth. We have used the ELMS procedures to provide a first-stage transformation and further research with other transformations would be useful, especially those which simultaneously adjust for skewness while fitting a multi-level model.

The application of multi-level models to fit the second stage of our procedure uses longitudinal data more efficiently than current methods ${ }^{5-7}$ especially when the data are unbalanced and incomplete. ${ }^{12,18}$ Our procedure is quite general for both univariate and multivariate data. Norms for any set of age intervals can be constructed and this overcomes a further important limitation 


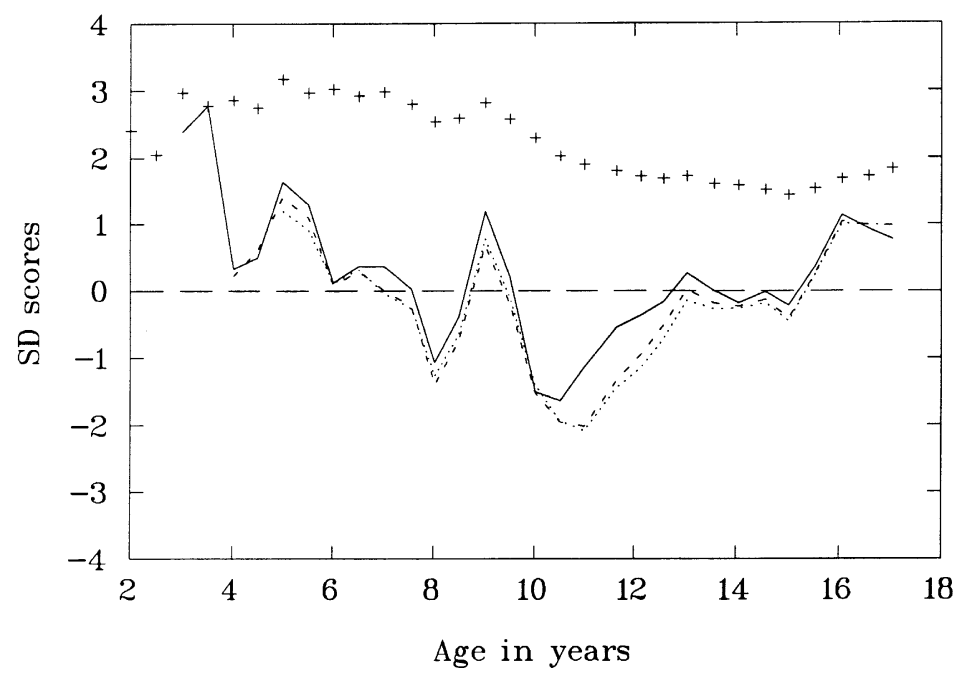

Figure 6. Standardized scores of conditional norms of weight for child 303

$++E L M S$ scores of weight

_ conditional on weight one year earlier

--- conditional on weight one and two years earlier

$\ldots$. conditional on weight one, two and three years earlier

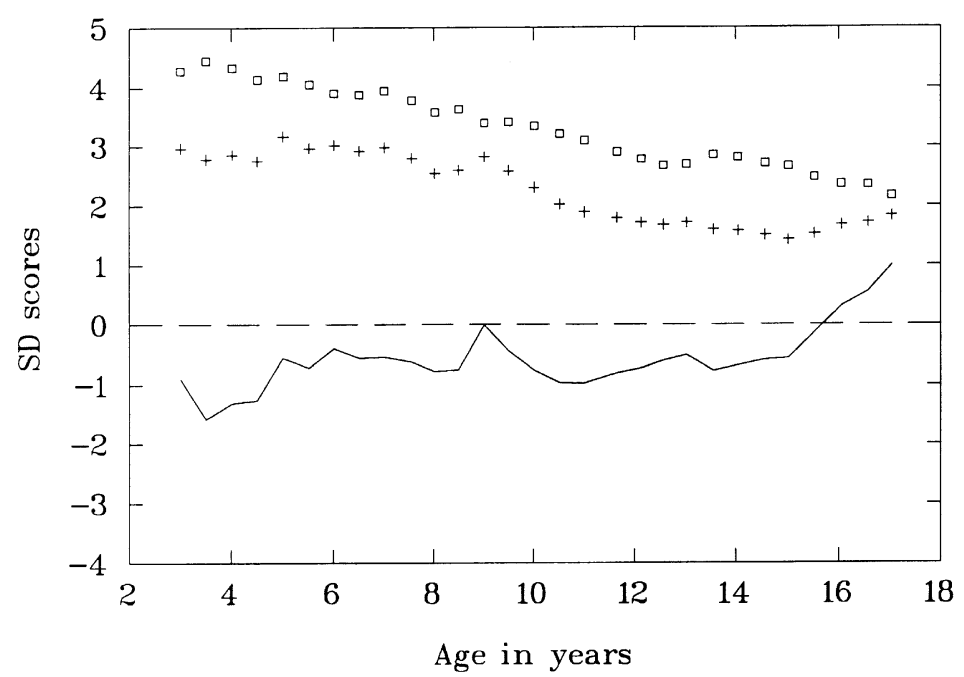

Figure 7. Standardized scores of weight conditional on height for child 303

$$
\begin{array}{ll}
\square \quad & \text { ELMS scores of height } \\
+\quad+ & \text { ELMS scores of weight } \\
& \text { conditional on height one year earlier }
\end{array}
$$




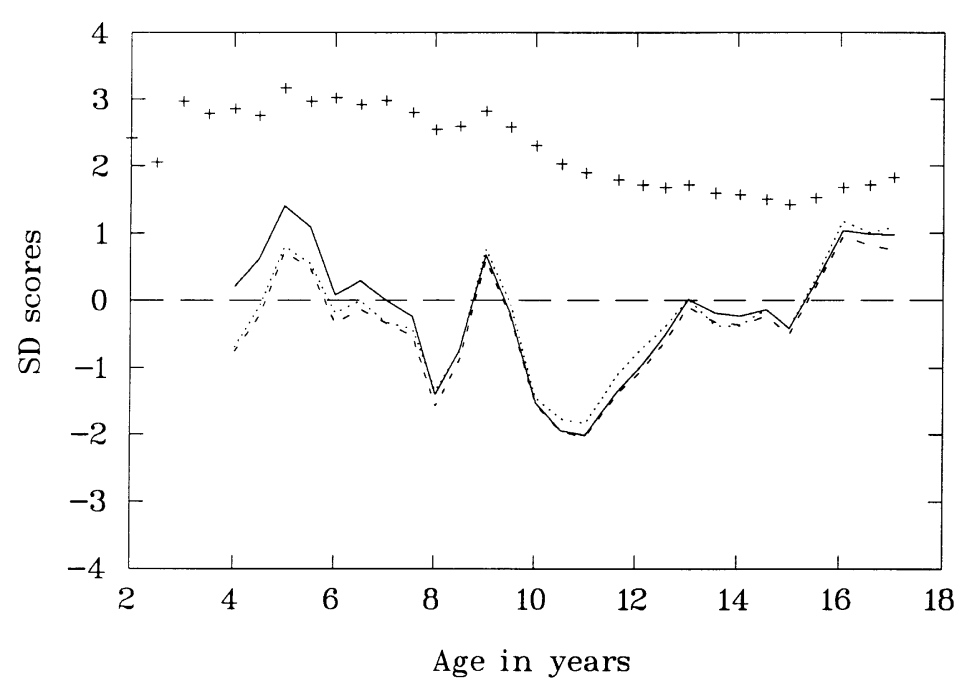

Figure 8. Standardized scores of weight conditional on weight and height for child 303

$+\quad+$ ELMS scores of weight

conditional on weight one and two years earlier

conditional on weight one and two years earlier and current height

conditional on weight one and two years earlier, current height and height one year earlier

of current procedures. For example, previous procedures for adjusting weight for height have used separate adjustments for each age group, although Cole ${ }^{17}$ presents an age-independent chart for age 6 to 12 years. In addition, our method makes it possible to derive the distribution of any function of measurements over time. Although we have not illustrated it, the method extends straightforwardly to incorporate covariates such as parental height ${ }^{2}$ and age-varying covariates such as family size.

Long term longitudinal data commonly contain only a few hundred individuals. Such a limited sample size is a problem in creating appropriate longitudinal norms for a population and our substantive results suffer from this limitation. It will be important, therefore, to replicate our findings on other populations. For the extreme percentiles our estimates are very sensitive to the correlation values; the 95 per cent Normal confidence interval for a correlation of 0.95 is $(0.934$, $0.962)$ with a sample of 200 measurements and is $(0.926,0.966)$ with a sample of 100 measurements. It is hence crucial to estimate correlations accurately using a large sample. For this reason also our present results should be regarded as provisional. Furthermore, we have chosen to use data covering a very wide age interval, but this strictly is unnecessary if interest centres only on one age range, for example adolescence.

There is a general problem of estimating the precision of our norms with small or moderate size samples. To do this we would need to take account of the precision of the parameter estimates at both stages of the procedure. Analytical results are unavailable and one approach is to carry out a bootstrap procedure, resampling individuals from the data set. Further work on this is planned.

Our results suggest that conditional norms using two or three previous measurements are very close together and are clearly different from those using just the previous measurement. This is 


\section{Intercept:}

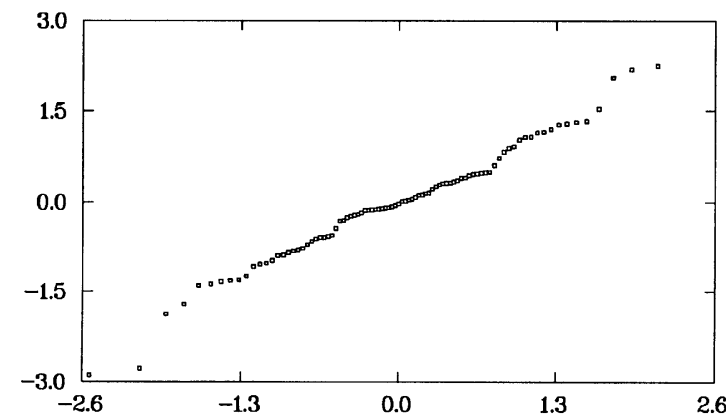

Slope:

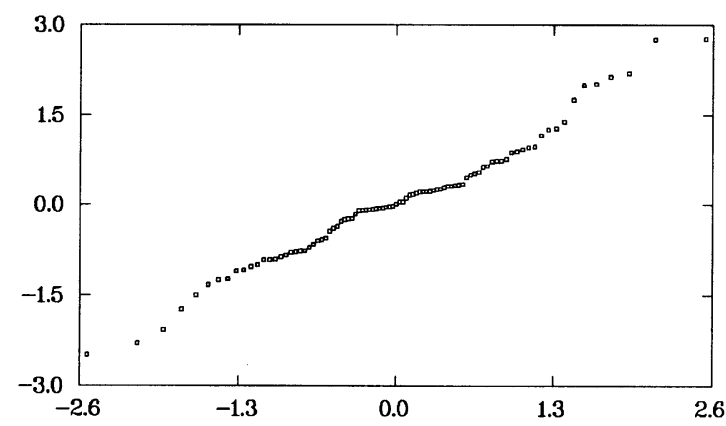

Level 1:

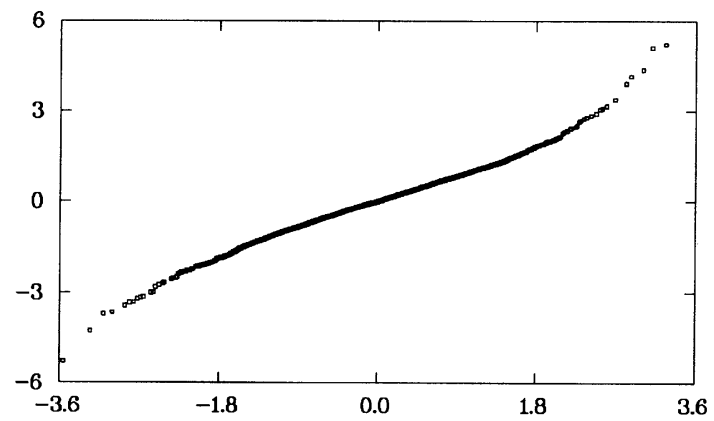

Figure 9. Standardized level 2 residuals and level 1 residual ( $y$ axis) plotted against Normal equivalent scores ( $x$ axis) for model (7) for height

consistent with the result of $\mathrm{Cole}^{7}$ so that the use of two previous one yearly measurements would seem to be recommended.

Because of the complexity of displaying the norms we have constructed, it is necessary to present these in a computing environment. We are developing personal computer software which will allow a user to input a set of measurements and ages and the appropriate estimated 
population norms to be displayed. The software will also allow users to supply their own normalized data or to modify existing norms.

\section{ACKNOWLEDGEMENTS}

We are grateful to John Rasbash for program assistance, and to Tim Cole for advice and help with the LMS estimates. We wish to acknowledge Dr. Shirley Ratcliffe for her permission to use the data from the Edinburgh Longitudinal Study. We also wish to thank Michael Healy, Michael Preece and Jim Tanner for helpful comments. We are also most grateful to referees for helpful comments. The work is supported by a grant from the Medical Research Council.

\section{REFERENCES}

1. Tanner, J. M., Whitehouse, R. H. and Takaishi, M. 'Standards from birth to maturity for height, weight, height velocity, and weight velocity: British Children, 1965, Archives of Disease in Childhood, 41, 454-471, 613-635 (1996).

2. Tanner, J. M., Goldstein, H. and Whitehouse, R. H. 'Standards for children's height at ages 2 to 9 years, allowing for height of parents', Archives of Disease in Childhood, 45, 755-762 (1970).

3. Tanner, J. M., Lejarrage, H. and Healy, M. J. R. 'Within-family standards for birthweight, a revision', Lancet, ii, 1314-1315 (1972).

4. Healy, M. J. R. 'Notes on the statistics of growth standards', Annals of Human Biology, 1, 41-46 (1974).

5. Cameron, N. 'Conditional standards for growth in height of British children from 5.0 to 15.99 years of age', Annals of Human Biology, 7, 331-337 (1980).

6. Berkey, C. S., Reed, R. B. and Valadian, I. 'Longitudinal growth standards for pre-school children', Annals of Human Biology, 10, 57-67 (1983).

7. Cole, T. J. 'Growth charts for both cross-sectional and longitudinal data', Statistics in Medicine, 13, 2477-2492 (1994).

8. Cole, T. J. and Green, P. J. 'Smoothing reference centile curves: the LMS method and penalised likelihood', Statistics in Medicine, 11, 1305-1319 (1992).

9. Cole, T. J. 'Fitting smoothed centile curves to reference data', Journal of the Royal Statistical Society, Series A, 151, 385-418 (1988).

10. Royston, P. 'Calculation of unconditional and conditional reference intervals for foetal size and growth from longitudinal measurements', Statistics in Medicine, 14, 1417-1436 (1995).

11. Wade, A. M. and Ades, A. E. 'Age-related reference ranges: significance tests for models and confidence intervals for centiles', Statistics in Medicine, 13, 2359-2367 (1994).

12. Goldstein, H. Multilevel Statistical Models, Edward Arnold, London, Wiley, New York, 1995.

13. Goldstein, H., Healy, M. J. R. and Rasbash, J. 'Multilevel time series models with applications to repeated measures data', Statistics in Medicine, 13, 1643-1655 (1994).

14. Ayatollahi, S. M. T. 'Age standardisation of weight-for-height in children using a unified Z-score method', Annals of Human Biology, 22, 151-162 (1995).

15. Ratcliffe, S. G. and Paul, N. Prospective Studies in Children with Sex Chromosome Aneuploidy, Alan. R. Liss, New York, 1986.

16. Rasbash, J. and Woodhouse, G. MLn Command Reference. Version 1.0, Institute of Education, London, 1995.

17. Cole, T. J. 'Constructing growth charts smoothed across time and space, in Hauspie, R., Lindgren, G. and Falkner, F. (eds), Essays on Auxology, presented to James Mourilyan Tanner, Castlemead, Welwyn Garden City, 1995, pp. 76-88.

18. Goldstein, H. 'Efficient statistical modelling of longitudinal data', Annals of Human Biology, 13, 129-141 (1986).

19. Goldstein, H. and Pan, H. 'Percentile smoothing using piecewise polynomials, with covariates', Biometrics, 48, 1057-1068 (1992). 